\title{
Endogenous formation of prostanoids in neonates with persistent pulmonary hypertension
}

\author{
P G KÜHL, ${ }^{*}$ R B COTTON, $\dagger$ H SCHWEER, ${ }^{*}$ AND H W SEYBERTH* \\ From the Departments of Paediatrics, ${ }^{*}$ University of Heidelberg, West Germany, and †Vanderbilt University, \\ Nashville, United States
}

SUMMARY Endogenous formation of thromboxane $\mathrm{A}_{2}$ and prostacyclin were evaluated in seven neonatates with persistent pulmonary hypertension by serial gas chromatographic mass spectrometric determination of their urinary metabolites dinor-thromboxane $B_{2}$ and dinor-6keto-prostaglandin $F_{1 \alpha}$, respectively. The patients were studied until their hypertension had resolved on clinical criteria. Urinary excretion of dinor-thromboxane $\mathrm{B}_{2}$ and dinor-6keto-prostaglandin $F_{1 \alpha}$ was increased when the persistent pulmonary hypertension was associated with group $B$ streptococcal $(n=2)$ and pneumococcal $(n=1)$ sepsis. Based on urinary metabolite excretion, endogenous formation of thromboxane $A_{2}$ and prostacyclin did not consistently differ from normal neonates in four patients with non-septic persistent pulmonary hypertension (hyaline membrane disease $(n=2)$, asphyxia, and meconium aspiration). These data suggest that thromboxane $\mathrm{A}_{2}$ is not a universal mediator of persistent pulmonary hypertension. It may, however, have a role in the pathophysiology of early onset group B streptococcal disease, and persistent pulmonary hypertension of other infectious aetiology. If these findings are confirmed by further studies, thromboxane synthetase inhibition or receptor antagonism may offer a potential therapeutic approach in neonates with persistent pulmonary hypertension associated with sepsis.

There is ample evidence in experimental animals to implicate the vasoconstrictor prostanoid thromboxane $A_{2}$ in the pathophysiology of acute pulmonary hypertension. ${ }^{1-5}$ These conclusions are based on the effect of pharmacological intervention with cyclooxygenase $^{12}$ and thromboxane synthetase inhibitors, ${ }^{3}$ thromboxane receptor antagonists, ${ }^{5}$ and the measurements of thromboxane metabolites in plasma, ${ }^{235}$ lung lymph, ${ }^{1}$ and urine. ${ }^{5}$ Persistent pulmonary hypertension of the neonate is an example of increased pulmonary vascular resistance in the neonatal period and has lead to the hypothesis that thromboxane $\mathrm{A}_{2}$ might be a modulator of pulmonary vascular tone in this condition. The aim of this study was to assess prostanoid biosynthesis in neonates with persistent pulmonary hypertension and healthy controls using urinary excretion rates of enzymatic metabolites of thromboxane $A_{2}$ (dinor-thromboxane $B_{2}$ ) and prostacyclin (dinor-6-keto-prostaglandin $F_{1 \alpha}$ ) as indices of endogenous prostanoid formation. ${ }^{6}$

\section{Patients and methods}

Between April and June 1985 at Vanderbilt University Hospital and between March 1985 and January 1988 at the University Children's Hospital, Heidelberg neonates who met the clinical criteria of persistent pulmonary hypertension were enrolled in the study on their first day of life. Patients were excluded from the study if they died early in the course of the disease so that an adequate urine collection could not be made.

Three infants born at full term and four preterm infants (two at Vanderbilt University Hospital, five at Children's Hospital, Heibelberg) were available for study. Clinical suspicion of persistent pulmonary hypertension was based on severe labile hypoxaemia disproportionate to the severity of pulmonary disease and confirmed either by contrast echocardiography, hyperoxic hyperventilation test, or the difference between preductal and postductal arterial oxygen pressure $($ table $){ }^{7}$ All patients were 
Table Clinical characteristics of patients with persistent pulmonary hypertension

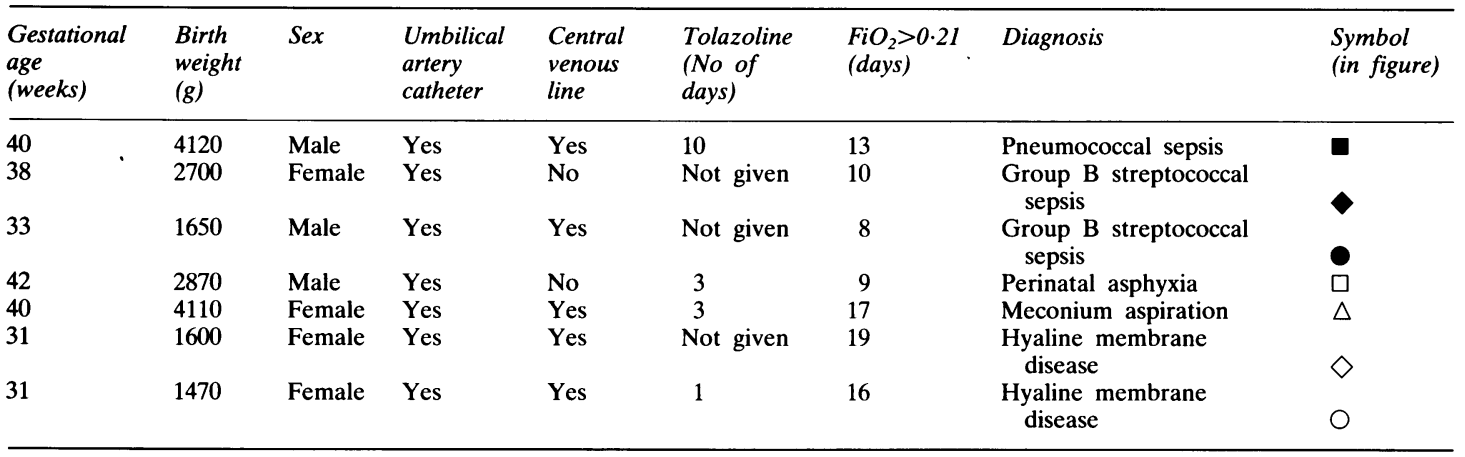

mechanically ventilated with a maximum fractional inspiratory oxygen $\left(\mathrm{FiO}_{2}\right)$ value cf 1.0 on day 1 . $\mathrm{FiO}_{2}$ requirements could be decreased to room air at a median of 13 (range 8-19) days. Mechanical ventilation was necessary for a median of 10 (range 5-19) days. Four of the infants were treated with tolazoline. One patient with meconium aspiration had an episode of thrombocytopenia, the lowest platelet count being $42 \times 10^{9} / 1$ on day seven. None of the babies died.

Urine was collected for 12 to 24 hours on two to seven occasions during the first 12 days of life until the persistent pulmonary hypertension had clinically resolved. Collection was made in self adhesive bags or from an indwelling catheter, if clinically indicated, and urine volume was determined. Analysis of dinor-thromboxane $B_{2}$ and dinor-6-keto-prostaglandin $F_{1 \alpha}$ by gas chromatography negative ion chemical ionisation mass spectrometry was carried out as described previously. ${ }^{8}$ Prostanoid metabolite excretion was expressed as $\mathrm{ng} / \mathrm{h} / 1.73 \mathrm{~m}^{2}$. The normal range of prostanoid metabolite excretion during the neonatal period was determined on 56 occasions in 29 neonates some born preterm and some at full term, using the same methods.

The study protocol was approved by the ethics committees of both hospitals.

\section{Results}

Excretion of the thromboxane metabolite dinorthromboxane $B_{2}$ varied considerably among the individual patients (fig 1). Whereas the neonates with persistent pulmonary hypertension associated with sepsis had excretion rates consistently above the normal range early in their course, the three other patients without sepsis (cases 4, 6, and 7 in the table) did not exceed control values. Case 5 (with severe perinatal asphyxia and meconium aspiration) had normal dinor-thromboxane $\mathrm{B}_{2}$ excretion at the

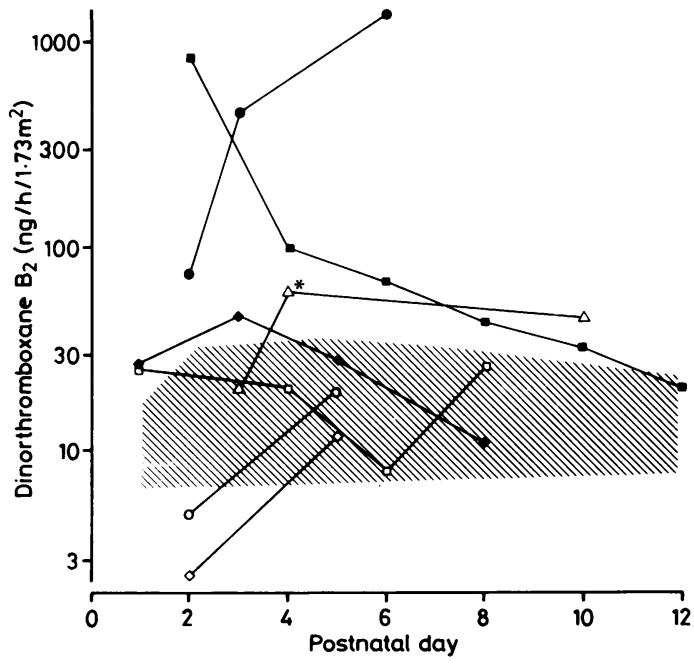

Fig 1 Urinary excretion of dinor-thromboxane $B_{2}$ (logarithmic scale). Symbols for each patient are given in the table. Shaded area $=$ normal range. ${ }^{*}$ Episode of thrombocytopenia.

peak of the disease, which increased as the thrombocytopenia developed, and the $\mathrm{FiO}_{2}$ went down to $0 \cdot 5$. Metabolite excretion reflected the clinical course-for example, the fraction of inspired oxygen as a global index of right to left shunting in cases 1 and 2 . Case 3, however, was unique in that his dinor-thromboxane $B_{2}$ excretion peaked at a time when the pulmonary hypertension had resolved clinically and oxygen requirements had decreased to room air. For this reason, no further follow up data are available.

The association between $\mathrm{FiO}_{2}$ requirements and the excretion rates of dinor-thromboxane $B_{2}$ is shown in fig 2 . There was no positive correlation 


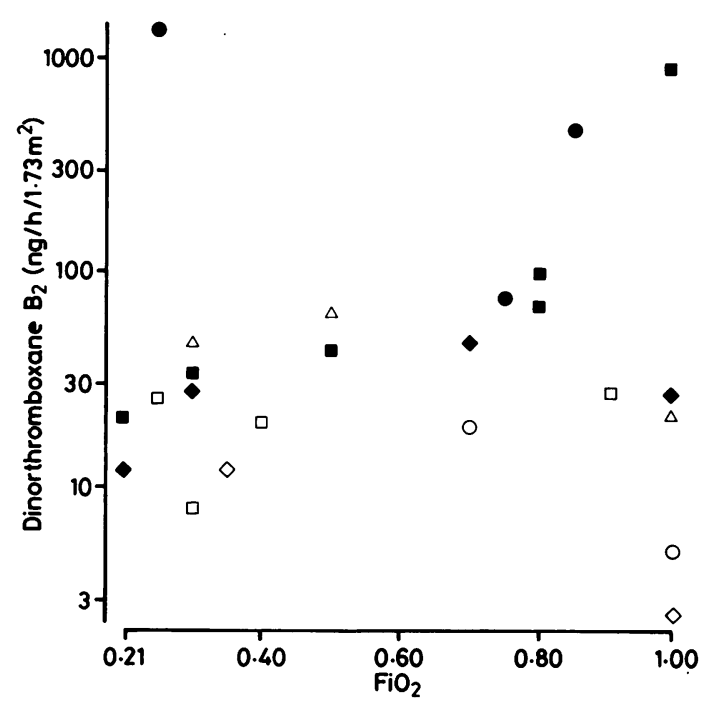

Fig 2 Relationship between $\mathrm{FiO}_{2}$ and excretion rates of dinor-thromboxane $B_{2}$ (logarithmic scale). Symbols for each patient are given in the table.

between these two variables in patients with persistent pulmonary hypertension not related to sepsis $(r=-0.39$ by linear regression analysis). In neonates in whom it was associated with sepsis, however, the two variables showed a trend towards positive correlation: linear regression analysis $r=0.33(0.05$ $<\mathrm{p}<0 \cdot 1)$.

Excretion rates of the prostacyclin metabolite dinor-6-keto-prostaglandin $\mathrm{F}_{1 \alpha}$ followed the general pattern of the thromboxane metabolite (fig 3).

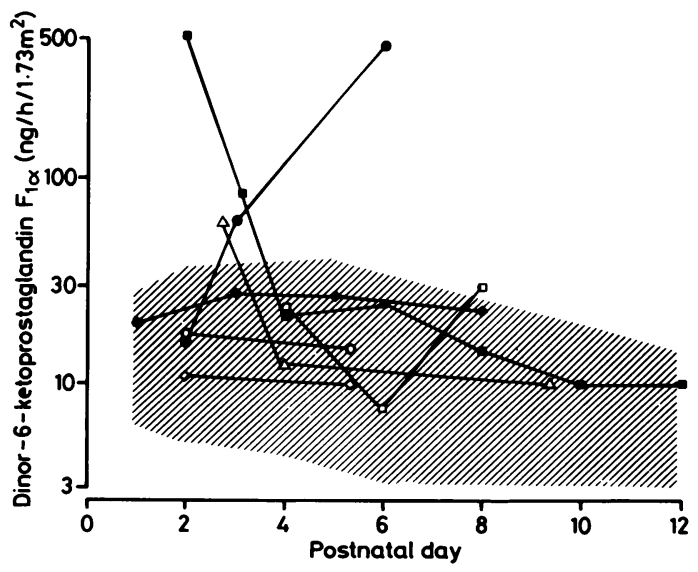

Fig 3 Urinary excretion of dinor-6-keto-prostaglandin $F_{l \alpha}$ (logarithmic scale). Symbols for each patient identification are given in the table. Shaded area=normal range.
Again, patients with pulmonary hypertension associated with sepsis excreted more dinor-6keto-prostaglandin $\mathrm{F}_{1 \alpha}$ than their non-septic counterparts.

Mean (SEM) urine output during the sampling periods was comparable in the two groups: $3 \cdot 3(0 \cdot 3)$ $\mathrm{ml} / \mathrm{kg} / \mathrm{h}$ for patients with hypertension associated with sepsis and $3.2(0.7) \mathrm{ml} / \mathrm{kg} / \mathrm{h}$ for those without sepsis. Thus differences in urine output do not account for variations in metabolite excretion.

\section{Discussion}

The present study showed that urinary metabolite excretion was consistently higher early in the course of pulmonary hypertension associated with sepsis compared with hypertension without sepsis. The two groups of patients resembled each other in many respects-for example, the degree of ventilator dependency, oxygen requirements, and the number of central venous and arterial lines. The latter factor has been shown to increase excretion of both the thromboxane and prostacyclin metabolites. ${ }^{59}$ The pattern of thromboxane and prostacyclin metabolite excretion, both in neonates with persistent pulmonary hypertension and normal neonates, agrees well with the results previously found in extremely low birthweight infants (less than $1000 \mathrm{~g}$ ), although more immature and probably sicker premature infants tend to have higher excretion rates. ${ }^{8}$ Serial determinations of metabolite excretion did not mirror the clinical courses in non-septic patients with persistent pulmonary hypertension, whereas an association with $\mathrm{FiO}_{2}$ requirements in pulmonary hypertension associated with sepsis is suggested. In the only patient with thrombocytopenia, thromboxane metabolite excretion reflected the decreasing platelet count rather than the course of pulmonary vasoconstriction, as shown by the oxygen dependency. Thus other factors-for example, platelet activation-have to be considered in the interpretation of increased thromboxane biosynthesis. Our results suggest that in persistent pulmonary hypertension, thromboxane $A_{2}$ is not apparently the only modulator of increased pulmonary vascular tone and that the relevance of thromboxane $\mathrm{A}_{2}$ depends on the aetiology of the pulmonary hypertension. These data support the hypothesis that thromboxane $\mathrm{A}_{2}$ may contribute to the pathophysiology of sepsis, as suggested by animal experiments. ${ }^{1-5}$

On the other hand, these results of urinary thromboxane metabolite excretion are at variance with increased immunoreactive plasma thromboxane $B_{2}$ reported in persistent pulmonary hypertension or predominantly non-infectious aetiology by Hammerman et al ${ }^{10}$ and Ford et al. ${ }^{11}$ Circulating 
plasma concentrations of thromboxane $B_{2}$ have been estimated in the $1-2 \mathrm{pg} / \mathrm{ml}$ range in adults by infusion studies. ${ }^{12}$ Although plasma concentrations may be higher in neonates, ${ }^{8}$ plasma concentrations in the $30-3000 \mathrm{pg} / \mathrm{ml}^{10}$ or even $10-60 \mathrm{ng} / \mathrm{ml}^{11}$ range most likely reflect in vivo platelet activation rather than genuine circulating thromboxane $B_{2}$ as an index of endogenous thromboxane $\mathrm{A}_{2}$ formation. ${ }^{6} 1314$ Likewise the differerence between normal controls and those with persistent pulmonary hypertension ${ }^{10}$ may mirror the fact that platelets in the latter may be more 'sticky' and easily activated, as shown by the incidence of thrombocytopenia in this study. The course of thromboxane metabolite excretion in our only thrombocytopenic patient is in agreement with this assumption. In the more standardised setting of an animal experiment, Hammerman et al ${ }^{15}$ were able to distinguish between pulmonary hypertension associated with sepsis and that associated with hypoxia on the basis of plasma thromboxane concentrations, which supports these data.

Any evaluation of endogenous thromboxane $A_{2}$ and prostacyclin formation in clinical studies should rely on a dependable analytical approach. ${ }^{16}$ In this respect, thromboxane $B_{2}$ and 6-ketoprostaglandin $F_{1 \alpha}$ in plasma are indices that may readily be confounded by in vivo artefacts. ${ }^{614} 16$ Urinary enzymatic metabolites have been shown to be useful analytical targets in various clinical studies. ${ }^{6} 1416$ Our data obtained by such an approach show that endogenous thromboxane and prostacyclin formation are increased early in the course of persistent pulmonary hypertension associated with sepsis. If experience with more patients should confirm these results, new treatment modalities by thromboxane synthase inhibitors or receptor antagonists might improve the prognosis of certain subtypes of persistent pulmonary hypertension. In view of the increase in endogenous prostacyclin formation, however, exogenous administration of this potent pulmonary vasodilator does not seem to be a logical therapeutic intervention in persistent pulmonary hypertension associated with sepsis.

This work was supported by the Deutsche Forschungsgemeinschaft (Se 263, Ku 544) and the National Institute of Health (HD 17413. HD 05797, and HL 14214). HW Seyberth is a Herrmann and Lilly Schilling Professor for Medical Research from the Stifterverband für die Deutsche Wissenschaft. We thank the nursing staff of the two hospitals for their help in collecting the urine specimens and our laboratory technicians for performing the prostanoid assays.

\section{References}

1 Rojas J, Larsson LE, Ogletree ML, et al. Effects of cyclooxygenase inhibition on the response to group B streptococcal toxin in sheep. Pediatr Res 1983;17:107-10.

2 Runkle B, Goldberg RN, Streitfeld MM, et al. Cardiovascular changes in group B streptococcal sepsis in the piglet: response to indomethacin and relationship to prostacyclin and thromboxane A. Pediatr Res 1984;18:874-8.

${ }^{3}$ Truog WE, Sorensen GK, Standaert TA, Redding GJ. Effects of the thromboxane synthetase inhibitor, dazmegrel (UK 38,485 ), on pulmonary gas exchange and hemodynamics in neonatal sepsis. Pediatr Res 1986;20:481-6.

${ }^{4}$ Brigham KL, Meyrick B. State of art: endotoxin and lung injury. Am Rev Respir Dis 1986;133:913-27.

5 Kühl PG, Bolds JM, Loyd JE, et al. Thromboxane receptor mediated bronchial and hemodynamic responses in ovine endotoxemia. Am J Physiol 1988;254:R310-9.

6 FitzGerald GA, Pedersen AK, Patrono C. Analysis of prostacyclin and thromboxane biosynthesis in cardiovascular disease. Circulation 1983;67:1174-7.

${ }^{7}$ Fox WW, Duara S. Persistent pulmonary hypertension in the neonate: diagnosis and management. J Pediatr 1983;103;505-14.

${ }^{8}$ Kühl PG, Cotton RB, FitzGerald GA. Systemic production of prostacyclin and thromboxane $A_{2}$ does not correlate with patency of the ductus arteriosus in very low birth weight infants. J Pediatr 1986;108:977-82.

9 Roy L, Knapp HR, Robertson RM, FitzGerald GA. Endogenous biosynthesis of prostacyclin during cardiac catheterization and angiography in man. Circulation 1985;71:434-40.

10 Hammerman C, Lass N, Strates E, et al. Prostanoids in neonates with persistent pulmonary hypertension. J Pediatr 1987;110: 470-2.

$"$ Ford WDA, James MJ, Walsh JA. Congenital diaphragmatic hernia: association between pulmonary vascular resistance and plasma thromboxane concentrations. Arch Dis Child 1984;59: 143-6.

12 Patrono C, Ciabattoni G. Pugliese F, et al. Estimated rate of thromboxane secretion into the circulation of normal man. J Clin Invest 1986;77:590-4.

13 Schweer H, Kammer J, Kühl PG, Seyberth HW. Determination of peripheral plasma prostanoid concentration: an unreliable index of 'in vivo' prostanoid activity. Eur J Clin Pharmacol 1986:31:303-5.

${ }^{14}$ FitzGerald GA, Healy C. Daugherty J. Thromboxane $A_{2}$ biosynthesis in human disease. Fed Proc 1987;46:154-8.

15 Hammerman C, Komar K. Abu-Khudair H. Hypoxic vs septic pulmonary hypertension. Am J Dis Child 1988:142:319-25.

16 Seyberth HW, Kühl PG. The role of eicosanoids in paediatrics. Eur J Pediatr 1988:147:341-439.

Correspondence to Dr P Gonne Kühl, Universitäts-Kinderklinik, Im Neuenheimer Feld 150, D-6900 Heidelberg, West Germany.

Accepted 15 February 1989 